\title{
Strength of Preference and Effects of Valence in the Domains of Gains and Losses
}

\author{
KIMIHIKO YAMAGISHI \\ Shukutoku University, Saitama, J apan
}

In comparative judgments that follow binary choices, judgments of "How much better is a preferred option?" and "How much worse is a less preferred option?" may differ in their magnitudes ("valence effects"). This paper analyzed cognitive processes that underlie positive valence effects ("Better" exceeding "Worse") and negative valence effects (Worse exceeding Better). My analyses used a "focus shift model." The focus shift model postulates that choice options are represented as sets of desirable and undesirable features. Difference judgments are reached by assigning subjective weights onto such features and integrating weighted feature contributions. Positive and negative valence effects reflect the differences in subjective weighting depending on valence of judgments. Experiments 1 through 3 showed that systematic positive valence effects were observed in the domain of gains, whereas negative valence effects were observed in the domain of losses. Estimates of subjective weights showed that valence effects occurred when partici pants heavily weighted desirable features in preferred options and undesirable features in less preferred options. These patterns of subjective weighting for positive and negative valence effects were consistent with the focus shift model. Data were more consistent with the focus shift model over alternative explanations of valence effects. Relationship with relevant economic and psychological phenomena are discussed.

The judgmental literature has repeatedly shown that logically equivalent magnitude judgments may differ

This work is based on the author's doctoral dissertation submitted to University of Washington. I thank J ohn Miyamoto (advisory committee chair), Richard Gonzalez, Earl Hunt, Elizabeth Loftus, and Deborah McCutchen for their encouragement, guidance, and criticism throughout my graduate career. The hel pful suggestions from two anonymous reviewers are gratefully acknowledged. My appreciation extends to Laura Kray and J ulie Feldman for their comments on an earlier draft. A preliminary report of this research was presented at the 34th annual meeting of The Psychonomic Society. Address correspondence and reprint requests to Kimihiko Yamagishi, Faculty of Business and Environment, Shukutoku University, Fujikubo 1150-1, Miyoshi-Cho, I ruma-Gun, Saitama 354, J apan. E-mail: kimihiko@shukutoku.ac.jp or PAG00457@niftyserve.or.jp. in their magnitudes depending on how the tasks are performed (e.g.: Banks, Clark, \& Lucy, 1975; Dunning \& Parpal, 1989; Lichtenstein \& Slovic, 1971; Lindman, 1971; Tversky, 1977). Such observations led researchers to question whether the discrepancies are due to changes in cognitive representation of the tasks, or due to changes in mental processes involved. I refer to disparities of this sort as asymmetries. Asymmetry is the main psychological issue of this paper.

Research on preferential choice provides a classic example of asymmetry. In preference reversal phenomena (Lichtenstein \& Slovic, 1971; Lindman, 1971), decision makers may prefer Gamble $P$ that offers higher probability and lower payoff than Gamble D, yet rate Gamble D more favorably in pricing of the Gambles. Also, Shafir (1993) demonstrated asymmetries between choosing and disowning. He presented a choice between two options, one of which consisted of moderately favorable and unfavorable features. Another option consisted of extremely favorable and extremely unfavorable features. Shafir's participants designated the extreme option when they were required to choose one out of two, but the same option was rejected when participants were required to assume the two in possession and disown either one. Research on similarity judgment provides another well-known example. Tversky's (1977) participants rated North Korea as more similar to China than China was to North Korea.

To explain such asymmetries, some theorists adopted the notion of feature-decomposition of objects of judgment, and task-dependent subjective weighting upon such features. In Tversky's case, the objects were decomposed into sets of shared and unique features. Similarity judgment is reached by assigning subjective weights to the feature sets, and by combining the weighted contribution of each feature to the judgment. The asymmetry arises when the subjective weighting to features that are unique to each object differs between judgments of "How similar is A to Z?" and "How similar is Z to A?" In preference reversals (Shafir, Osherson, \& Smith, 1989; Tversky, Sattath, \& Slovic, 1988; Tversky, Slovic, \& Kahneman, 1990), the payoff and probability of each gamble 
constitute the features in choice options. The preference for a higher-probability gamble in binary choice is explained by decision makers assigning heavier subjective weights to probabilities, whereas the higher price for the high-payoff gamble in bidding (which implies a reversal of preferential order) is explained by participants assigning heavier subjective weights to payoffs. In the choice-rejection asymmetry (Shafir, 1993), subjective weights are assigned to sets of desirable and undesirable features in choice options. In choice, decision makers assign heavier weights to desirable features, thereby the option with extremely desirable features is chosen. Conversely, in rejection, decision makers assign heavier weights to undesirable features, thereby the option with extremely undesirable features is disowned.

This paper extends this line of research in the following sense. I introduce asymmetries in comparative difference judgments, and theories on such asymmetries based on feature-weighting approach. The review indicates the phenomenon of interest in this paper, namely an asymmetry in strength-of-preference judgments.

\section{MENTAL ADDITION AND SUBTRACTION (DUNNING \& PARPAL, 1989)}

Dunning and Parpal noted an asymmetry in comparative judgments that involved counterfactual reasoning. They asked undergraduates at their first choice college to compare their college and the other second choice college that they would have attended if the first choice rejected their application (the first choice and the second choice were clearly recognized by each participant). One group of participants was asked to rate how much more challenging their classes at First Choice College are than they would have been at Second Choice College. The other group was asked to rate how much less challenging their courses would have been at Second Choice than they are at First Choice. The magnitude judgments in this comparison showed that the former $(M=6.0)$ was reliably greater than the latter $(M=3.4)$. Their numerous replications showed that judgments of How much more produced greater magnitudes than judgments of How much less. The Dunning-Parpal asymmetry was discovered in this sense.

Dunning and Parpal explained their findings by what they termed Mental Addition and Subtraction. The mental addition was defined as "assessments in which people must determine whether a causal agent will produce an outcome to a greater degree" (Dunning \& Parpal, 1989, p. 5). The mental subtraction was defined by replacing the word "greater" with "lesser." Specifically, they adopted the notions of feature-decomposition and task-dependent changes in subjective weights. In the First Choice vis-à-vis Second Choice comparison, each school has relevant features that contribute to judgment of the quality of education. I could be assumed that the contribution for the positive impacts (e.g., quality of education) would be stronger at the First Choice. In answering the "How much better at the First Choice" question, subjective weights would be more heavily assigned to the First Choice, because it is the subject of comparison in the particular question phrase. J udgments reached by such a procedure result in assigning stronger subjective weights to features with stronger contributions. In contrast, in answering the "How much worse at the Second Choice" questions, the subject of comparison now switches to the Second Choice. Heavier subjective weights would be assigned to the Second Choice. Hence, relatively weaker weights are assigned to features with the stronger contribution in the First Choice, whereas stronger weights are assigned to features with lesser contributions at the Second Choice. The Dunning-Parpal asymmetry occurs because, by the mental addition, subjective weighting stretches the differences in feature contributions between the superior and inferior options of choice, whereas by the mental subtraction, the subjective weights are assigned such that, when the feature contributions are summed up to reach a judgment, the heavy weighting of the inferior option decreases the gap between the differences between the superior and inferior options in their feature contributions. For example, suppose that the qualities of education at the First and the Second choice are 8 and 6, respectively. Suppose further that the weights for the subject of comparison and the alternative choice option are 2 and 1 , respectively. Assuming a process of weighted linear combination, the mental addition would produce a different judgment of $8 \times 2-6 \times 1=10$. In turn, the mental subtraction would produce a difference of $6 \times$ $2-8 \times 1=4$. Thus, mental addition exceeds mental subtraction.

The Dunning-Parpal asymmetry inspired subsequent research. Dhar and Simonson (1992) applied the idea of subjective-weight shifts among the subjects of comparisons to analyze consumer preferences. Business major students were asked to assume that they had been admitted to business schools at Harvard and Stanford and to express their preference. Prior to indicating their choice, one group of participants was required to compare the schools in terms of how much more attractive Harvard was and how much they preferred Harvard more. The response scale was positive in favor of Harvard (positive numbers if Harvard was more attractive/preferable) and negative in disfavor of Harvard (negative numbers if Harvard was less attractive/preferable). The other group was required to compare the same schools, yet the subject of comparison 
Option A

(Preferred)

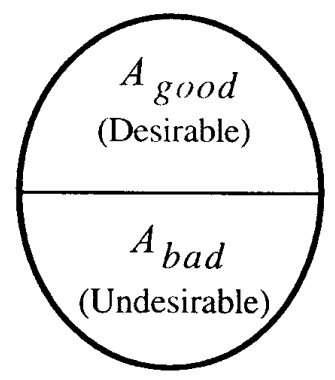

FIG. 1. The feature-set representation of choice options in the focus shift model.

was changed to Stanford. Each group rated the subjectof-comparison school more favorably, and, in each group, the majority of participants preferred that school. Dhar and Simonson argued that the subject of comparison attracted heavier subjective weighting, and the weighting influenced decision makers' articulation of preferential orders.

\section{VALENCE EFFECTS AND FOCUS SHIFT MODEL (YAMAGISHI \& MIYAMOTO, 1996)}

As in Dhar and Simonson, the Dunning-Parpal asymmetry led Yamagishi and Miyamoto (1996) to investigate a similar asymmetry. Yamagishi and Miyamoto found that judgments of "How much better is a preferred option?" and "How much worse is a less preferred option?" may differ in their magnitudes. They called the former and the latter types of judgment as superiority judgment and inferiority judgment, respectively. They called it a positive valence effect when a superiority judgment exceeds an inferiority judgment for an identical pair of options, and a negative valence effect for the difference in the opposite direction. To analyze the cognitive process that produced such asymmetries, Yamagishi and Miyamoto proposed a focus shift model. Like many feature-weighting models, the focus shift model is built upon feature-decomposition of choice options, and task-dependent subjective weighting plays a central role to explain positive and negative valence effects.

The focus shift model applies to comparative judgments between Options $A$ and $Z$, where $A$ is preferred to $Z$. These Options are represented as unique sets of features, and the features are either desirable or undesirable. Figure 1 shows the set representation and terminology for the feature sets in the focus shift model.

The focus shift model represents superiority and inferiority judgments by Formulas (1) and (2), respectively:

$$
\begin{aligned}
& D_{\mathrm{s}}=\alpha_{\mathrm{s}} \mathrm{f}\left(\mathrm{A}_{\text {good }}\right)-\beta_{\mathrm{s}} \mathrm{f}\left(\mathrm{A}_{\text {bad }}\right)-\left[\gamma_{\mathrm{s}} \mathrm{f}\left(\mathrm{Z}_{\text {good }}\right)-\eta_{\mathrm{s}} \mathrm{f}\left(\mathrm{Z}_{\text {bad }}\right)\right] \\
& \mathrm{D}_{\mathrm{i}}=\alpha_{\mathrm{i}} \mathrm{f}\left(\mathrm{A}_{\text {good }}\right)-\beta_{\mathrm{i}} \mathrm{f}\left(\mathrm{A}_{\text {bad }}\right)-\left[\gamma_{\mathrm{i}} \mathrm{f}\left(\mathrm{Z}_{\text {good }}\right)-\eta_{\mathrm{i}} \mathrm{f}\left(\mathrm{Z}_{\text {bad }}\right)\right]
\end{aligned}
$$

Subscripts s and i stand for superiority and inferiority, respectively. $\mathrm{D}$ represents difference judgment, and $\alpha$, $\beta, \gamma$, and $\eta$ denote the subjective weighting that are associated with corresponding feature sets. $f(\cdot)$ represents the contribution of each feature set to the judgment. It follows from Formulas (1) and (2) that a positive valence effect is observed when $D_{s}>D_{i}$, iff

$$
\begin{aligned}
& \left(\alpha_{\mathrm{s}}-\alpha_{\mathrm{i}}\right) \mathrm{f}\left(\mathrm{A}_{\text {good }}\right)-\left(\beta_{\mathrm{s}}-\beta_{\mathrm{i}}\right) \mathrm{f}\left(\mathrm{A}_{\text {bad }}\right) \\
& \quad>\left(\gamma_{\mathrm{s}}-\gamma_{\mathrm{i}}\right) \mathrm{f}\left(\mathrm{Z}_{\text {good }}\right)-\left(\eta_{\mathrm{s}}-\eta_{\mathrm{i}}\right) \mathrm{f}\left(\mathrm{Z}_{\mathrm{bad}}\right)
\end{aligned}
$$

and a negative valence effect is observed when $D_{s}<$ $D_{i}$, iff

$$
\begin{aligned}
& \left(\alpha_{\mathrm{s}}-\alpha_{\mathrm{i}}\right) \mathrm{f}\left(\mathrm{A}_{\mathrm{good}}\right)-\left(\beta_{\mathrm{s}}-\beta_{\mathrm{i}}\right) \mathrm{f}\left(\mathrm{A}_{\mathrm{bad}}\right) \\
& \quad<\left(\gamma_{\mathrm{s}}-\gamma_{\mathrm{i}}\right) \mathrm{f}\left(\mathrm{Z}_{\mathrm{good}}\right)-\left(\eta_{\mathrm{s}}-\eta_{\mathrm{i}}\right) \mathrm{f}\left(\mathrm{Z}_{\mathrm{bad}}\right)
\end{aligned}
$$

Note that Formulas (3) and (4) postulate that positive and negative valence effects occur when subjective weighting changes as a function of valence of the question. The label, "focus shift," reflects the idea of such weighting changes. Yamagishi and Miyamoto used hypothetical gamble pairs that varied in payoffs, and one gamble (Option A) al ways dominated the other (Option Z). They found that, for gambles that consisted of payoffs of winning and losing outcomes, participants were equally likely to produce positive and negative valence effects (i.e., a group of participants systematically produced positive valence effects, whereas another group systematically produced negative valence effects). They used regression analysis to estimate the subjective weighting parameters for each participant. For each subjective weight, they calculated the difference between superiority and inferiority judgments (e.g., for $\mathrm{A}_{\text {good }}$, they calculated $\alpha_{\mathrm{s}}-\alpha_{\mathrm{i}}$ ). For the participants who produced positive valence effects, the differences of subjective weights followed the pattern of boxplots in the top panel of Fig. 2. The top panel of Fig. 2 shows that positive valence effects were associated with the $\alpha$ and $\eta$ differences that were greater than zero, whereas the $\beta$ and $\gamma$ differences were closer to zero. The middle panel of Fig. 2 shows the pattern of estimated differences in subjective weights for the group of participants who produced reliable negative valence effects (the distinction between the black versus dotted boxes, as well as the interpretation of the bottom panel of Fig. 2 , will be explained in relation with the hypothesis of Experiment 2). The top and the middle panels show mirror images of each other, along the zero line. From these results, Yamagishi and Miyamoto argued that it 

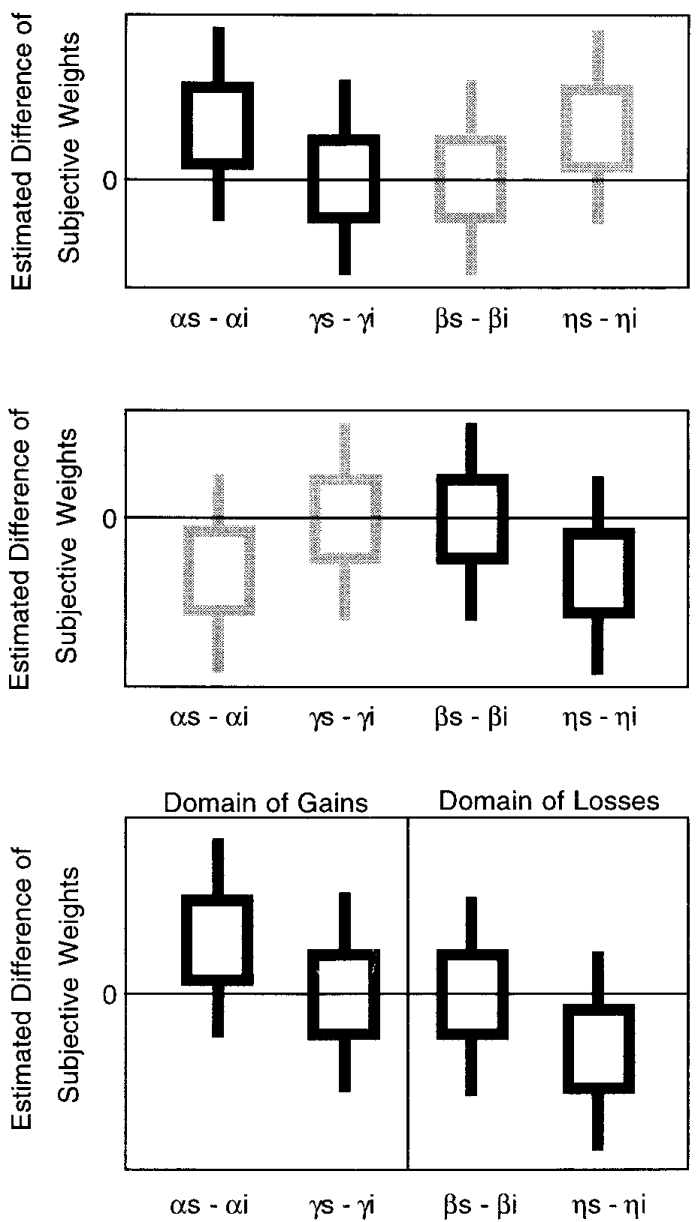

FIG. 2. Subjective weighing patterns observed in a previous study (top and middle panels, Yamagishi \& Miyamoto, 1996) and predicted for Experiment 2 (bottom).

was subjective weights on $A_{\text {good }}$ and $Z_{\text {bad }}$ that produced systematic valence effects. The participants with heavy subjective weights on these feature sets in superiority judgments (i.e., $\alpha_{\mathrm{s}}$ and $\eta_{\mathrm{s}}$ were great) produced the $\alpha$ and $\eta$ differences scattering above the zero line, and Formula (3) tends to hold from this weighting pattern. In contrast, the participants with heavy subjective weights on $A_{\text {good }}$ and $Z_{\text {bad }}$ in inferiority judgments (i.e., $\alpha_{\mathrm{i}}$ and $\eta_{\mathrm{i}}$ were great) produced the $\alpha$ and $\eta$ differences scattering below zero, and Formula (4) tends to hold. The $\beta$ and $\gamma$ differences scatter along the zero line, suggesting little focus shift concerning these two feature sets ( $A_{\text {bad }}$ and $\left.Z_{\text {good }}\right)$.

Moreover, Yamagishi and Miyamoto (1996) documented that the directions of valence effects at the aggregate level were predictable from the intrinsic pleasantness of the stimulus domain. In another experiment, they sampled choice options from intrinsically pleasant domains (e.g., vacation plans) and from intrinsically unpleasant domains (e.g., painful treatments for cancer). When choice options were sampled from pleasant domains, the mean superiority and inferiority ratings exhibited positive valence effects. Conversely, when choice options were chosen from unpleasant domains, participants exhibited reliable negative valence effects. For instance, in a choice between vacation plans, the mean superiority and inferiority ratings were 10.48 and 7.42, respectively. In a choice between painful treatments for cancer, the mean superiority and inferiority ratings were 9.33 and 10.60, respectively.

\section{THE PURPOSE OF THIS PAPER}

This paper extends the line of research set forth by Yamagishi and Miyamoto by investigating two psychological issues that remain to be explored. First, although Yamagishi and Miyamoto (1996) showed that the directions of valence effects varied depending on the intrinsic pleasantness of the stimulus domains, the patterns of subjective weighting under such conditions remain to be uncovered. Second, the focus shift model will be compared to a class of other plausible models to examine their empirical agreement with data. The following experiments investigate these issues.

Experiment 1 aimed at replicating the results from Yamagishi and Miyamoto (1996) using stimuli chosen from a semantically simpler domain, i.e., gambles. Experiment 2 was designed to estimate the subjective weighting parameters under systematic positive and negative valence effects to verify whether the weighting pattern is consistent with the prediction derived from the results from Yamagishi and Miyamoto (1996). Experiment 3 contrasts the focus shift model and other plausible models that may explain valence effects. These rival models were compared with respect to an empirical relationship among choice options that must hold if the alternative models were in effect.

\section{EXPERIMENT 1}

The purpose of Experiment 1 is to examine whether the pattern of valence effects in intrinsically pleasant versus unpleasant domains with complex stimuli (Yamagishi \& Miyamoto, 1996) is also obtained using monetary gambles. Hypothetical gambles with either exclusively winning payoffs or exclusively losing payoffs were used as stimuli. These two sets of gambles represent a pleasant and unpleasant domain, respectively. It is predicted that positive valence effects occur with exclusively winning gambles, whereas negative valence effects occur with exclusively losing gambles. The rationale for using gambles is as follows: Gambles with either exclusively winning or exclusively losing payoffs leave little variability for individual differences in the 
interpretation of choice options as desirable or undesirable. In addition, gambles with explicitly stated payoff and probability have been used as the standard choice alternatives in decision making research (for a review, see Slovic, Lichtenstein, \& Fischhoff, 1988).

\section{Method}

Participants. Participants were 187 University of Washington undergraduates. They were enrolled in an introductory psychology course and participated to earn extra credit.

Variables. Two independent variables were used. One was the domain of choice options, consisting of gain and loss conditions. These conditions were represented by exclusively winning and exclusively losing gambles, respectively. Another independent variable was valence of judgment (either superiority or inferiority). The dependent variables were superiority and inferiority rating on a 16-point scale that ranged from 0 (no difference) to 15 (maximum difference).

Stimuli. Appendix A shows the pairs of gambles used in Experiment 1. In each stimulus pair, the payoffs of Option A were set to be higher than those of Option Z, to maintain the relation that A was always preferable to Z. Exclusively losing gambles were generated by changing payoffs from "Win" to "Lose."

Design and prediction. A mixed design was used. The domain was a between-subject variable; 94 participants were assigned to the gain condition and 93 participants were assigned to the loss condition. The valence was a within-subject variable. It was predicted that positive valence effects would be observed in the gain condition, whereas negative valence effects would be observed in the loss condition.

Procedure. All the experiments reported in this paper followed the procedure outlined here, except for minor differences (such as the amount of payoffs) that are introduced whenever necessary. Data were gathered in group settings. Each participant was given a booklet of gamble choices that contained other filler tasks. Participants were instructed that they could choose either Gamble A or Z, and the outcome was determined by flipping a fair coin (i.e., a 50-50 chance between Heads and Tails). The gambles were hypothetical and no actual monetary transactions occurred in the experiment. For each gamble pair, participants were first asked to express their preferences. Subsequently, they evaluated the gamble in terms of "How much better is the option that you chose than the option that you did not choose?" and "How much worse is the option that you did not choose than the option that you chose?" The order of gamble presentation, as well as answering "Better" and "Worse" questions, were randomized and counterbalanced across participants.

\section{Results and Discussion ${ }^{1}$}

All participants preferred the dominant option at every opportunity for choice (the same tendency was observed in Experiments 2 and 3, thus will not be mentioned repeatedly hereafter). For each gamble pair, I performed a 2 by 2 ANOVA (domain by valence) to estimate the error term $\left(\mathrm{MS}_{\mathrm{e}}\right)$ for two orthogonal planned comparisons. The [ $1-100]$ contrast, denoting the contrast values for the superiority-gain, inferioritygain, superiority-loss, and inferiority-loss conditions, tested for positive valence effects in the gain condition. Likewise, the [ $\left[\begin{array}{llll}0 & 0 & 1 & -1\end{array}\right]$ contrast tested the negative valence effects in the loss condition. The MS s for Gambles 1 through 4 were 6.64, 5.98, 8.44, and 7.13, respectively ( $\mathrm{df}=185$ ). Table 1 shows the mean superiority and inferiority judgments for the gain and loss conditions. The t values in the gain and loss conditions were calculated by subtracting the mean inferiority judgment from the mean superiority judgment. Therefore, a positive $t$ value indicates a positive valence effect, whereas a negative t value indicates a negative valence effect.

Table 1 shows empirical supports for the prediction. In the gain condition, reliable positive valence effects were found for all gamble pairs. Conversely, in the loss condition, the direction of valence effects was all reliably negative. It may be concluded that Experiment 1 replicated the findings of Yamagishi and Miyamoto (1996), using hypothetical gambles as choice options.

\section{EXPERIMENT 2}

Although the results from Experiment 1 were consistent with Yamagishi and Miyamoto's (1996) findings, the results alone do not necessarily indicate whether focus shift occurred when positive and negative valence effects were observed. Therefore, it is desirable to estimate subjective weight parameters $\left(\alpha_{\mathrm{s}}, \alpha_{\mathrm{i}}, \beta_{\mathrm{s}}, \beta_{\mathrm{i}}, \gamma_{\mathrm{s}}\right.$, $\gamma_{\mathrm{i}}, \eta_{\mathrm{s}}$, and $\eta_{\mathrm{i}}$ ) to confirm that they do change as a function of valence, and that the pattern of weight change is consistent with the logic of the focus shift model. Experiment 2 was designed to estimate subjective weighting parameters under positive valence effects in pleasant domains and negative valence effects in unpleasant domains.

\footnotetext{
${ }^{1}$ Two-tailed tests were used in all data analyses in this paper. In text and tables, *,**, and *** are used to denote a statistical significance at the $.05, .01$, and .001 level, respectively.
} 
TABLE 1

Mean Rating and $t$ Test Statistic for Experiment 1

\begin{tabular}{cccccccc}
\hline & \multicolumn{3}{c}{ Gain condition } & & \multicolumn{3}{c}{ Loss condition } \\
\cline { 2 - 3 } Pair & Superiority & Inferiority & $\mathrm{t}(185)$ & & Superiority & Inferiority & $\mathrm{t}(185)$ \\
\hline 1 & 12.86 & 11.07 & $6.66^{* * *}$ & & 11.28 & 11.97 & $-2.57^{* *}$ \\
2 & 11.44 & 9.46 & $7.76^{* * *}$ & & 10.06 & 10.72 & $-2.59^{* *}$ \\
3 & 14.95 & 13.13 & $6.01^{* * *}$ & & 13.40 & 14.55 & $-3.80^{* * *}$ \\
4 & 13.43 & 11.27 & $7.75^{* * *}$ & & 11.55 & 12.73 & $-4.24 * * *$ \\
\hline
\end{tabular}

\section{Prediction for the Subjective Weighting Pattern}

Given the results from Experiment 1, it would be reasonable to predict that positive valence effects would be observed in intrinsically pleasant domains. From the subjective weighting pattern noted by Yamagishi and Miyamoto (1996), the U-shaped pattern in the top panel of Fig. 2 corresponds to positive valence effects. However, in Yamagishi and Miyamoto's experiments, the intrinsic pleasantness of their stimuli was unclear a priori. Therefore, the focus shift model consisted of four feature subsets, $A_{\text {good }}, A_{\text {bad }}$, $Z_{\text {good, }}$, and $Z_{\text {bad }}$. In turn, when the focus shift model is applied to intrinsically pleasant domains, it could be assumed that the sets of undesirable features contribute very little to judgments. Therefore, el iminating the $A_{\text {bad }}$ and $Z_{\text {bad }}$ boxes from the top panel of Fig. 2 leaves the black boxes as the predicted subjective weighting pattern for pleasant domains. Formally, positive valence effects were described by Formula (3). Assuming that the contributions of $A_{\text {bad }}$ and $Z_{\text {bad }}$ are negligible in pleasant domains (i.e., $f\left(A_{\text {bad }}\right) \approx$ $\left.f\left(Z_{\text {bad }}\right) \approx 0\right)$, deletion of these terms leave the following reduced focus shift model to describe positive valence effects in the domain of gains:

$$
\begin{array}{r}
D_{s}>D_{i} \text { iff } \quad\left(\alpha_{s}-\alpha_{i}\right) f\left(A_{\text {good }}\right) \\
-\left(\gamma_{s}-\gamma_{i}\right) f\left(Z_{\text {good }}\right)>0 .
\end{array}
$$

If $f\left(A_{\text {good }}\right)>f\left(Z_{\text {good }}\right)$ is assumed, the inequality in Formula (5) follows from the pattern in the black boxplot (the $\alpha$ difference is very positive and greater than the $\gamma$ difference).

Conversely, in intrinsically unpleasant domains, negative valence effects would be predicted, given the observation in Experiment 1. Moreover, Yamagishi and Miyamoto (1996) observed the $\cap$-shaped pattern as in the middle panel of Fig. 2 when negative valence effects occurred. Assuming that $A_{\text {bad }}$ and $Z_{\text {bad }}$ are the only relevant feature sets in representing stimuli from unpleasant domains, deleting the dotted boxes from the middle panel of Fig. 2 leads to the prediction for subjective weighing in the domain of losses, as represented by the black boxes. In such cases, the focus shift model for negative valence effects in Formula (4) reduces to Formula (6) by presuming that $f\left(A_{\text {good }}\right) \approx f\left(Z_{\text {good }}\right) \approx 0$,

$$
D_{s}<D_{i} \text { iff } \quad\left(\eta_{s}-\eta_{i}\right) f\left(Z_{\text {bad }}\right)
$$

$$
-\left(\beta_{\mathrm{s}}-\beta_{\mathrm{i}}\right) \mathrm{f}\left(\mathrm{A}_{\text {bad }}\right)<0 .(6)
$$

Again, if one assumes that $f\left(Z_{\text {bad }}\right)>f\left(A_{\text {bad }}\right)$, the inequality in Formula (6) follows from the pattern in the black boxplot (the $\eta$ difference is very negative compared to the $\beta$ difference).

Considering only the black boxes from the top and middle panels of Fig. 2, the bottom panel shows the predicted patterns of subjective weights for the domains of gains and losses. The $\alpha$ difference tends to be very positive, whereas the $\eta$ difference tends to be very negative. The $\beta$ and $\gamma$ differences are predicted to scatter around the zero line.

\section{Testing for the Necessity of the Weight Parameters}

As discussed above, the explanation of valence effect using the focus shift model requires eight parameters, namely $\alpha_{s}, \alpha_{i}, \beta_{s}, \beta_{i}, \gamma_{s}, \gamma_{i}, \eta_{s}$, and $\eta_{i}$. Having to allow for the eight parameters may seem lacking in parsimony and el egance. One way to evaluate the necessity of these parameters in explaining the phenomenon would be to generate models that constrain some of the parameters to be equal, and compare the fits between the focus shift model and such other models.

Recall that the focus shift model reduced for intrinsically pleasant domains is:

$$
D_{s}=\alpha_{s} f\left(A_{\text {good }}\right)-\gamma_{s} f\left(Z_{\text {good }}\right)
$$

and

$$
D_{i}=\alpha_{i} f\left(A_{\text {good }}\right)-\gamma_{i} f\left(Z_{\text {good }}\right)
$$


Likewise, in intrinsically unpleasant domains, the re duced model is:

$$
D_{s}=\eta_{s} f\left(Z_{\text {bad }}\right)-\beta_{s} f\left(A_{\text {bad }}\right)
$$

and

$$
D_{i}=\eta_{i} f\left(Z_{\text {bad }}\right)-\beta_{i} f\left(A_{\text {bad }}\right)
$$

The first constraint I propose is to inhibit the focus shift to occur, namely to constrain the weights for superiority and inferiority judgments to be equal. Formulas (11) and (12) represent such models for desirable and undesirable domains, respectively.

$$
D \cdot=\alpha \cdot f\left(A_{\text {good }}\right)-\gamma \cdot f\left(Z_{\text {good }}\right)
$$

and

$$
D \cdot=\eta \cdot f\left(Z_{\text {bad }}\right)-\beta \cdot f\left(A_{\text {bad }}\right)
$$

where the constraints are that $\mathrm{D}_{\mathrm{s}}=\mathrm{D}_{\mathrm{i}}=\mathrm{D} \cdot, \alpha_{\mathrm{s}}=\alpha_{\mathrm{i}}$ $=\alpha \cdot$, and similarly for $\beta, \gamma$, and $\eta$.

Another constraint would be to force the subjective weights for the preferred and less preferred options to be equal. Thus, Formulas (7) and (8) would be rewritten as:

$$
D_{s}^{\prime}=\kappa_{s}\left[f\left(A_{\text {good }}\right)-f\left(Z_{\text {good }}\right)\right]
$$

and

$$
D_{i}^{\prime}=\kappa_{i}\left[f\left(A_{\text {good }}\right)-f\left(Z_{\text {good }}\right)\right]
$$

Likewise, Formulas (9) and (10) would be rewritten as:

$$
D_{s}^{\prime}=\lambda_{s}\left[f\left(Z_{\text {bad }}\right)-f\left(A_{\text {bad }}\right)\right]
$$

and

$$
D_{i}^{\prime}=\lambda_{i}\left[f\left(Z_{\text {bad }}\right)-f\left(A_{\text {bad }}\right)\right]
$$

The models in Formulas (13) through (16) assume that $\alpha_{\mathrm{s}}=\gamma_{\mathrm{s}}=\kappa_{\mathrm{s}}, \alpha_{\mathrm{i}}=\gamma_{\mathrm{i}}=\kappa_{\mathrm{i}}$, and similarly for $\beta, \eta$, and $\lambda$.

Lastly, the final constraint requires that the weights for the preferred and less preferred option, as well as superiority and inferiority ratings, be equal. Thus, imposing to Formulas (11) and (12) that $\alpha \cdot=\gamma \cdot=\pi$ and $\beta \cdot=\eta \cdot=\theta$, one obtains,

$$
D \cdot{ }^{\prime}=\pi\left[f\left(A_{\text {good }}\right)-f\left(Z_{\text {good }}\right)\right]
$$

and

$$
D \cdot{ }^{\prime}=\theta\left[f\left(Z_{\text {bad }}\right)-f\left(Z_{\text {bad }}\right)\right]
$$

for the domains of gains and losses, respectively.

The top and the bottom panels in Fig. 3 show a scheme of the hierarchical relationship among these models, for the domains of gains and losses, respectively. Implications among the models are indicated by arrows in the hierarchy. One of the purposes in Experiment 2 was to fit the models in Formulas (7) through (18) for each participant's responses to compare the goodness of fit among such models.

As in Experiment 1, Experiment 2 also used hypothetical gambles. A major difference from Experiment 1 lies in a factorial design with more levels in the payoffs in dominating and dominated gambles, and in the use of a completely within-subject design. Such a setting enabled to examine the directions of val ence effects and to estimate subjective weight parameters for each participant.

Method

Participants. Thirty-one University of Washington undergraduates participated to earn extra credit for an introductory psychology course.

Design. A within-subject design was used. Independent variables were domain (gains or losses), valence (superiority or inferiority), and amount of payoffs in dominating and dominated options (4 by 4). Dependent variables were judgments of superiority and inferiority on a 16-point scale ranging from 0 (no difference) to 15 (maximum difference).

Stimuli. Hypothetical two-outcome gambles were used as stimuli, which are listed in Appendix B. Let Sets $A$ and $Z$ denote sets of exclusively winning gambles (gain condition), where each el ement in Set $A$ dominates each element in Set Z. Set A consisted of Gambles $\{b, c, d, e\}$ and Set $Z$ consisted of gambles $\{v, w, x, y\}$. In contrast, let Sets $A^{\prime}$ and $Z^{\prime}$ denote sets of exclusively losing gambles (loss condition). Stimuli in the loss condition were generated by changing the payoffs from Sets $A$ and $Z$ from winning to losing. Set $A^{\prime}$ consisted of Gambles $\left\{b^{\prime}, c^{\prime}, d^{\prime}, e^{\prime}\right\}$ and Set $Z^{\prime}$ consisted of Gambles $\left\{v^{\prime}, w^{\prime}, x^{\prime}, y^{\prime}\right\}$. Again, every element in Set $A^{\prime}$ dominated every element in Set $Z^{\prime}$.

Procedure The whole experiment consisted of two sessions. Each session consisted of two blocks. Between sessions, there was a 1-week interval. Each participant was randomly presented with either gain or loss condition at each session, and the order of the domain was counterbalanced between sessions. Between the two blocks, each participant worked on filler tasks. Each 


\section{Domain of Gains}

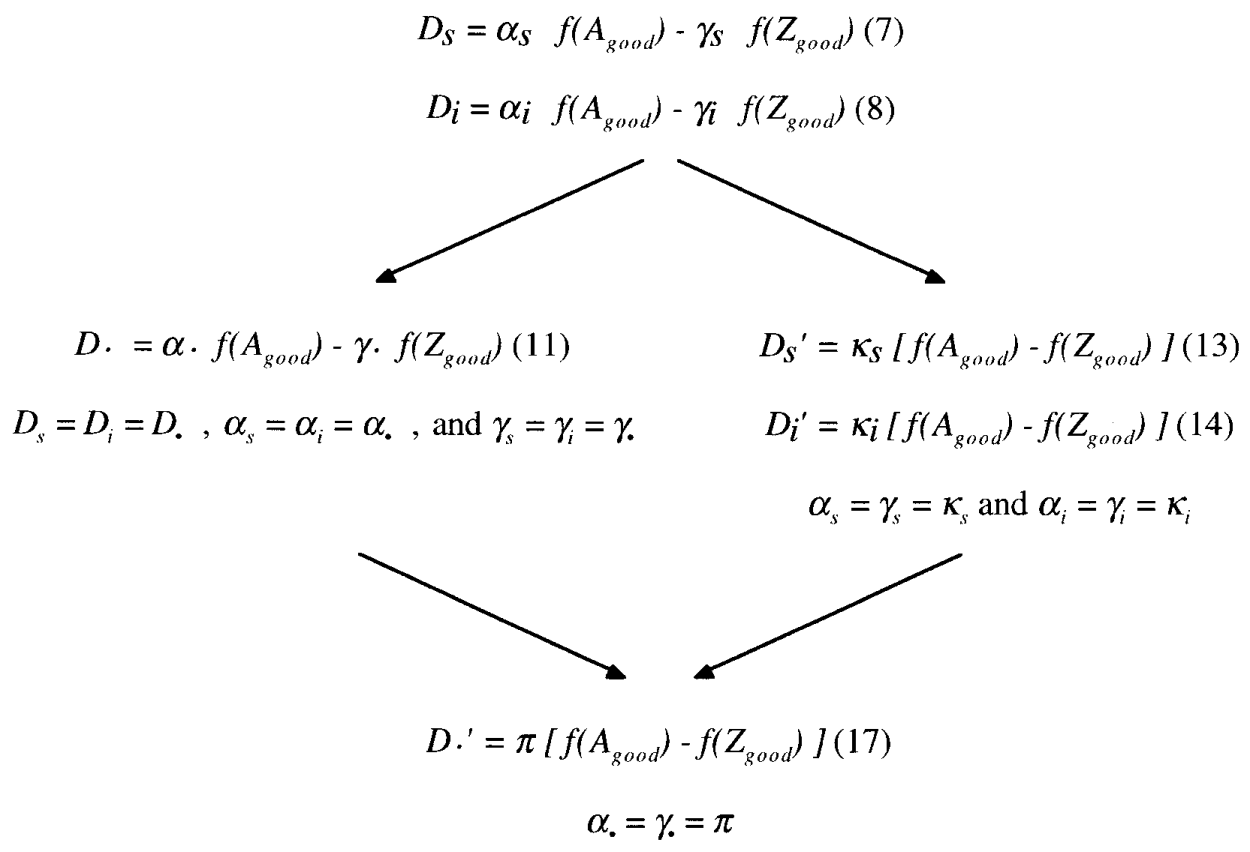

\section{Domain of Losses}

$D_{S}=\eta_{S} f\left(Z_{b a d}\right)-\beta_{S} f\left(A_{b a d}\right)(9)$

$D_{i}=\eta_{i} f\left(Z_{b a d}\right)-\beta_{i} f\left(A_{b a d}\right)(10)$
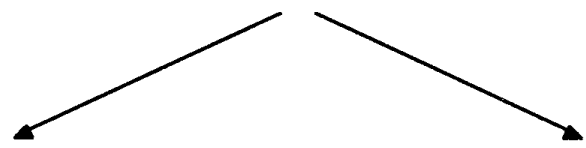

$D \cdot=\eta \cdot f\left(Z_{b a d}\right)-\beta \cdot f\left(A_{b a d}\right)(12)$

$D_{S}^{\prime}=\lambda_{S}\left[f\left(Z_{b u d}\right)-f\left(A_{b a d}\right)\right](15)$

$D_{s}=D_{i}=D_{.}, \beta_{s}=\beta_{i}=\beta_{.}$, and $\eta_{s}=\eta_{i}=\eta$.

$D_{i}^{\prime}=\lambda_{i}\left[f\left(Z_{b a d}\right)-f\left(A_{b a d}\right)\right](16)$

$\beta_{s}=\eta_{s}=\lambda_{s}$ and $\beta_{i}=\eta_{i}=\lambda_{i}$

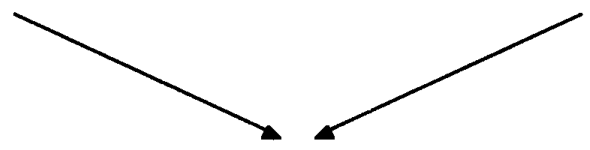

$$
\begin{gathered}
D .^{\prime}=\theta\left[f\left(Z_{b a d}\right)-f\left(A_{b a d}\right)\right] \\
\beta .=\eta_{\circ}=\theta
\end{gathered}
$$

FIG. 3. The hierarchical relationship among the models in Formulas (7) through (18).

participant was presented with either gain or loss condition across the blocks. The orders of gamble presentation and providing superiority and inferiority ratings were randomized and counterbalanced across participants.

\section{Results and Discussion}

For each participant's responses on each stimulus pair, the mean rating was calculated between the two blocks. Ther efore, 16 mean superiority ratings and 16 
TABLE 2

The Mean Superiority Rating, Inferiority Rating, and t Statistic at Aggregate Level in Experiment 2 (Gain Condition)

\begin{tabular}{|c|c|c|c|}
\hline Gamble pair & Superiority & Inferiority & $t(30)$ \\
\hline [b v] & 10.194 & 8.000 & $5.43 * * *$ \\
\hline$[\mathrm{b} w]$ & 9.887 & 7.807 & $5.84 * * *$ \\
\hline$[\mathrm{b} x]$ & 9.516 & 7.548 & $5.66 * * *$ \\
\hline [b y] & 8.790 & 6.807 & $5.25^{* * *}$ \\
\hline$[\mathrm{c} \mathrm{v}]$ & 10.919 & 8.677 & $4.83 * * *$ \\
\hline$[\mathrm{c} \mathrm{w}]$ & 10.581 & 8.177 & $5.97 * * *$ \\
\hline$[\mathrm{c} x]$ & 10.177 & 7.710 & $6.53 * * *$ \\
\hline [c y] & 9.565 & 7.210 & $6.00^{* * *}$ \\
\hline$[d \mathrm{v}]$ & 12.516 & 9.523 & $6.17 * * *$ \\
\hline$[d \mathrm{w}]$ & 11.823 & 8.742 & $7.05^{* * *}$ \\
\hline$[\mathrm{d} x]$ & 12.323 & 8.532 & $10.18 * * *$ \\
\hline [d y] & 10.936 & 7.903 & $8.05^{* * *}$ \\
\hline [e v] & 12.710 & 9.710 & $6.92^{* * *}$ \\
\hline [e w] & 12.645 & 9.177 & $7.45^{* * *}$ \\
\hline$[\mathrm{e} x]$ & 12.661 & 8.758 & $7.88 * * *$ \\
\hline [e y] & 12.855 & 8.419 & $10.23 * * *$ \\
\hline
\end{tabular}

mean inferiority ratings were obtained from every participant. Subsequent analyses wereall applied on these mean ratings.

Valence effects at aggregate level. For each gamble pair, the mean superiority and inferiority rating averaging across participants were calculated separately for the gain and the loss conditions. The mean difference was tested by a paired t test. Hereafter, as a notation, pairs of options are presented in brackets. For instance, $a[b \quad y]$ pair consists of Option b from Set $A$ and Option y from Set $Z$. Table 2 shows the mean superiority and inferiority ratings, as well as t test statistics $(\mathrm{df}=30)$ in the gain condition. For all gamble pairs, reliable positive valence effects were observed.

Table 3 shows the mean superiority ratings, mean inferiority ratings, and t-statistics for the loss condition. Most of the gamble pairs showed reliably negative valence effects. The exceptions were pairs $\left[b^{\prime} x^{\prime}\right]$ and $\left[d^{\prime} v^{\prime}\right]$, with the former pair showing a non-significant positive valence effect. I $n$ both gain and loss conditions, no reliable valence effect toward the opposite direction from the prediction was observed. Thus, the results at the aggregate level replicated Experiment 1 that positive valence effects were observed in the domain of gains, whereas negative valence effects were observed in the domain of losses.

Valence effects exhibited by individual participants. F or each participant, the differences between mean superiority and inferiority ratings were tested by a paired t test with $15 \mathrm{df}$. The type I error rate was controlled for 2 (gain and loss conditions) $\times 31(\mathrm{~N})$ comparisons. In the gain condition, 30 participants produced positive valence effects, of which 25 reached significance at the .05 level, as predicted. One participant produced a negative nonsignificant valence effect.

In the loss condition, mostly consistent with the prediction, 25 participants produced negative valence effects. Of those, 16 reached statistical significance at the .05 level. There was one case of significant positive valence effect. No participant produced a set of results toward the entirely opposite directions from the prediction, namely a significantly negative valence effect in the gain condition, AND a significantly positive valence effect in the loss condition. It may be claimed that, at the individual participant's level, data showed the predicted directions of valence effects in the gain and loss conditions.

Examining the necessity for the eight parameters. For each participant, I fitted the models in Formulas (7) through (18) using multiple regression. Because each model in Formulas (7) through (10) and (13) through (16) distinguish $D_{s}$ from $D_{i}$, a linear regression model was fitted to 16 data points separately for the superiority and inferiority ratings. In contrast, the models in Formula (11), (12), (17), and (18) do not distinguish superiority and inferiority ratings. Thus, these models were fitted to 32 data points collapsing over the superiority and inferiority ratings. For each data point, the corresponding payoffs for "Heads" for gambles from Set $A$ and $Z$ were used as the values of $f(\cdot)$. The payoffs for "Tails" were not incorporated in this procedure because they were perfectly correlated with the payoffs for Heads. Because these models differ in their degrees of freedoms that contributed to estimate the subjective weights parameters, adjusted $\mathrm{R}^{2}$ was used as a measure of comparison among these models.

\section{TABLE 3}

The Mean Superiority Rating, Inferiority Rating, and t Statistic at Aggregate Level in Experiment 2 (Loss Condition)

\begin{tabular}{crrr}
\hline Gamble pair & Superiority & Inferiority & $\mathrm{t}(30)$ \\
\hline$\left[\mathrm{b}^{\prime} \mathrm{v}^{\prime}\right]$ & 8.710 & 9.645 & $-2.40^{*}$ \\
{$\left[\mathrm{~b}^{\prime} \mathrm{w}^{\prime}\right]$} & 9.887 & 10.581 & $-2.16^{*}$ \\
{$\left[\mathrm{~b}^{\prime} \mathrm{x}^{\prime}\right]$} & 12.307 & 11.823 & .24 \\
{$\left[\mathrm{~b}^{\prime} \mathrm{y}^{\prime}\right]$} & 9.952 & 12.129 & $-4.56^{* * *}$ \\
{$\left[\mathrm{c}^{\prime} \mathrm{v}^{\prime}\right]$} & 7.597 & 9.065 & $-3.92^{* * *}$ \\
{$\left[\mathrm{c}^{\prime} \mathrm{w}^{\prime}\right]$} & 8.677 & 9.839 & $-2.82^{* *}$ \\
{$\left[c \mathrm{x}^{\prime}\right]$} & 9.177 & 11.097 & $-4.43^{* * *}$ \\
{$\left[\mathrm{c}^{\prime} \mathrm{y}^{\prime}\right]$} & 9.839 & 11.823 & $-4.28^{* * *}$ \\
{$\left[\mathrm{~d}^{\prime} \mathrm{v}^{\prime}\right]$} & 8.371 & 9.081 & -1.98 \\
{$\left[\mathrm{~d}^{\prime} \mathrm{w}^{\prime}\right]$} & 8.048 & 9.339 & $-3.54^{* *}$ \\
{$\left[\mathrm{~d}^{\prime} \mathrm{x}^{\prime}\right]$} & 8.807 & 11.548 & $-5.74^{* * *}$ \\
{$\left[\mathrm{~d}^{\prime} \mathrm{y}^{\prime}\right]$} & 9.613 & 11.968 & $-5.28^{* * *}$ \\
{$\left[\mathrm{e}^{\prime} \mathrm{v}^{\prime}\right]$} & 6.936 & 7.629 & $-2.33^{*}$ \\
{$\left[\mathrm{e}^{\prime} \mathrm{w}^{\prime}\right]$} & 7.597 & 8.629 & $-3.17^{* *}$ \\
{$\left[\mathrm{e}^{\prime} \mathrm{x}^{\prime}\right]$} & 7.378 & 10.484 & $-6.92^{* * *}$ \\
{$\left[\mathrm{e}^{\prime} \mathrm{y}^{\prime}\right]$} & 8.565 & 12.048 & $-6.35^{* * *}$ \\
\hline
\end{tabular}




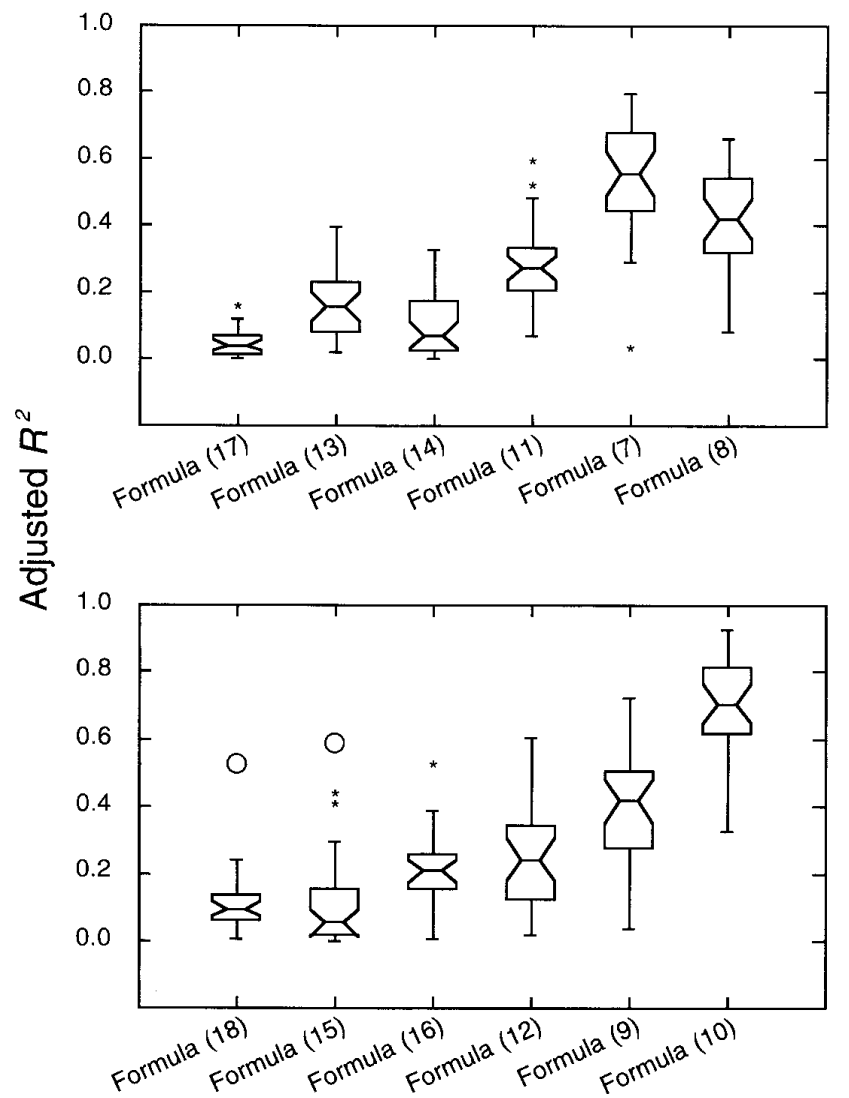

FIG. 4. Notched boxplots of adjusted $R^{2}$ for the judgmental models in the domain of gains (top) and losses (bottom).

The top panel of Fig. 4 shows notched boxplots ${ }^{2}$ (McGill, Tukey, \& Larsen, 1978) of the adjusted R ${ }^{2} \mathrm{~S}$ for each participant's responses, fitted by the models of judgments in the domain of gains. Note that the $R^{2} S$ are highest for Formula (7), followed by Formula (8). Other models that impose constraints on $\alpha_{\mathrm{s}}, \alpha_{\mathrm{i}}, \gamma_{\mathrm{s}}$, and $\gamma_{i}$ did not produce comparable fits, as shown by the boxplots for Formulas (11), (13), (14), and (17). Friedman's statistic was calculated to test the null hypothesis that the adjusted $\mathrm{R}^{2} \mathrm{~S}$ among the models differ due to chance alone. ${ }^{3}$ The null hypothesis was rejected, $\chi_{\mathrm{r}}^{2}$ $=123.63^{* * *}, \mathrm{df}=5$.

The bottom panel of Fig. 4 shows notched boxplots of adjusted $\mathrm{R}^{2}$ for each participant's responses, fitted

\footnotetext{
${ }^{2}$ In notched boxplots, the top and bottom ends of "whiskers" extend to the maximum and minimum observations excluding outliers. Outliers appear as open circles and asterisks. The upper and lower ends of the box represent the 75th and 25th percentiles, whereas the horizontal bar inside the box denotes the median. The notches that extend from the median indicate the $95 \%$ confidence bounds for the median.

${ }^{3}$ Friedman's nonparametric test was used because the pattern in Fig. 4 was suggestive of violations of assumptions for parametric tests.
}

by the models of judgments in the domain of losses. Again, the models that allow $\beta_{\mathrm{s}}, \beta_{\mathrm{i}}, \eta_{\mathrm{s}}$, and $\eta_{\mathrm{i}}$ to vary (Formulas (9) and (10)) achieve better fitting over other models that constrain these parameters. Friedman's statistic rejected the null hypothesis that the adjusted $\mathrm{R}^{2} \mathrm{~S}$ differ by chance alone, $\chi_{\mathrm{r}}^{2}=111.50 * * *$, $\mathrm{df}=5$. Thus, it may be argued that having the eight parameters for the focus shift model (Formulas (7) through (10)) achieves descriptive advantage over other models that allow for fewer parameters.

Estimating subjective weighting parameters. To estimate $\alpha_{\mathrm{s}}$ and $\gamma_{\mathrm{s}}$, F ormula (7) was fitted to each participant's superiority ratings in the gain condition. Likewise, to estimate $\alpha_{i}$ and $\gamma_{i}$, Formula (8) was fitted to the inferiority ratings in the gain condition. To estimate $\beta_{\mathrm{s}}$, $\eta_{\mathrm{s}}$, and $\beta_{\mathrm{i}}, \eta_{\mathrm{i}}$, Formulas (9) and (10) were fitted to the superiority and inferiority ratings, respectively, in the loss condition. Finally, the difference between each feature set's subjective weights was estimated by subtracting the weight estimate for the superiority ratings from the estimate for the inferiority ratings.

Figure 5 shows notched boxplots of the estimated differences of the subjective weights. Notice the resemblance of the predicted pattern in the bottom panel of Fig. 2 . In the gain condition, the $95 \%$ confidence intervals for the $\alpha$ differences lie above the zero line. However, the $\gamma$ differences scatter around the zero line, with the median close to zero. Formula (5) shows that the inequality for positive valence effects tends to hold with this weighting pattern. Recall that the dependent variables did exhibit systematic positive valence effects (Table 2). In contrast, the $95 \%$ confidence intervals for the $\beta$ and $\eta$ differences lie below the zero line, with the $\eta$ differences scattered further away. This pattern is obtained using the same data that showed systematic negative valence effects (Table 3). Thus, Fig. 5 shows

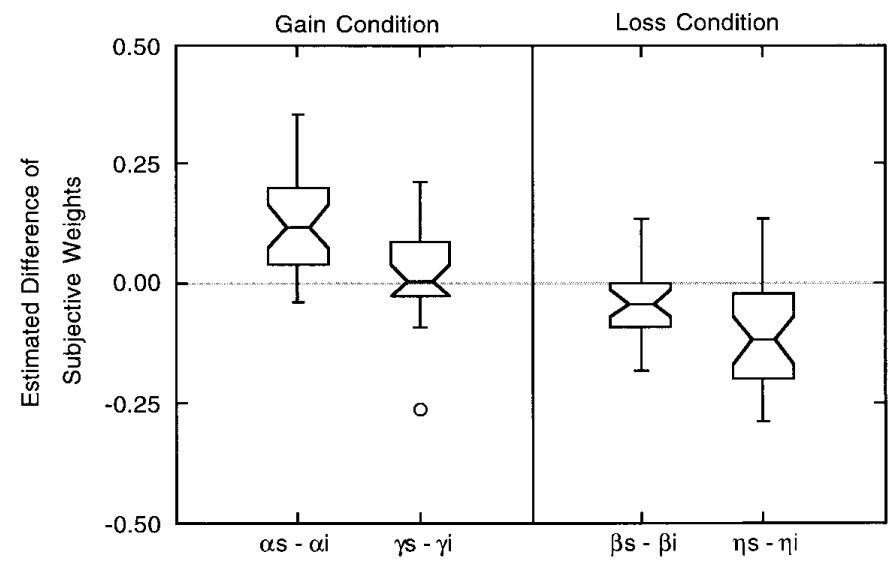

FIG. 5. Notched boxplots of estimated differences of subjective weights. 
that the subjective weighting parameters confirmed the prediction that followed from Yamagishi and Miyamoto's (1996) results, and were consistent with the description of positive and negative valence effects by the reduced focus shift models (Formulas (5) and (6)).

\section{EXPERIMENT 3}

The purpose of Experiment 3 is to compare the focus shift model to other alternative explanations that could explain valence effects by their agreement with data. The following section specifies a condition under which such alternative accounts and the focus shift model lead to different predictions. Experiment 3 was specifically designed to compare these rival models under such a condition. To illustrate the condition, the logic of the alternative models is discussed first.

Alternativemodels of valence effects. First, let the following notation hold: Suppose that Option i is preferred to Option $p$; Option $\mathrm{j}$ is preferred to Option q; and $\phi(\cdot)$ represents a value function for such options. An alternative model would claim that $D_{s}(i, p)=$ $M[\phi(i) / \phi(p)]$ whereas $D_{i}(i, p)=M^{\prime}[\phi(i) / \phi(p)]$. Such a model postulates that difference judgment is a ratio function between psychological values of Options $i$ and p. Another possibility would be to represent difference judgments as a function of proportional change. A valence effect could be found when $D_{s}(i, p)=M\{\phi(i)-$ $\phi(p) y_{\phi}(p)$, whereas $D_{i}(i, p)=M\{\phi(i)-\phi(p)\} \phi(i)$. These terms do not necessarily coincide because the former and latter formulas reduce to $\mathrm{M}[\phi(\mathrm{i}) / \phi(\mathrm{p})-1]$ and $\mathrm{M}[1-\phi(\mathrm{p}) / \phi(\mathrm{i})]$, respectively. In gist, these alternative arguments claim that positive and negative valence effects differ in the integration of psychological values for the preferred and the less preferred options. For instance, the latter argument suggests that positive valence effects reflect the sensitivity to the value increment of the preferred option over the less preferred option, whereas negative valence effects reflect the sensitivity to decrement of the less preferred option from the preferred option.

Such alternative models to the focus shift model must satisfy ordinal independence over infinite pairs of options (see Chapter 6 in Krantz, Luce, Suppes, \& Tversky, 1971, for details of this argument). Ordinal independence (hereafter referred to as OI ) is the following relation among pairs of choice options:

$$
D_{s}(i, p)>D_{s}(j, q) \text { iff } D_{i}(i, p)>D_{i}(j, q) \text {. }
$$

In words, OI requires that the superiority and inferiority judgments be order-preservative. Therefore, the rank-ordering relationship among pairs of options in

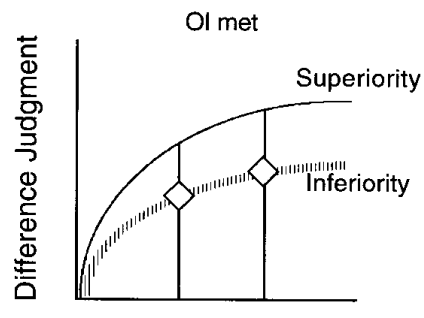

[j q] [i p]

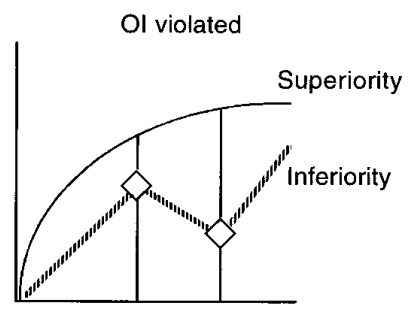

[j q] [i p]
FIG. 6. Hypothetical examples of adherence to (left) and violation of (right) OI.

superiority judgments should be the same as the ordering in inferiority judgments.

The left panel of Fig. 6 shows an illustration of a hypothetical result that adheres to OI. Suppose that one obtains superiority and inferiority ratings of a set of options, rank-orders them by the superiority ratings, and plots them. Furthermore, assume that, for each option, the corresponding inferiority ratings were overlaid on the pl ot of the superiority ratings. The left panel shows that both superiority and inferiority ratings should exhibit monotonic increase patterns. In addition, the square plot symbols indicate that the rankordering among the superiority ratings (i.e., $\left.D_{s}(i, p)>D_{s}(j, q)\right)$ is preserved in the inferiority ratings (i.e., $D_{i}(i, p)>D_{i}(j, q)$ ) as well.

In contrast, the focus shift model assumes that subjective weighting differs as a function of the valence of judgment. Formally, if the weights differ as a function of valence, then the observed rank-ordering of difference judgments over infinite option pairs must violate OI.

Moreover, the findings so far allow for specific predictions regarding the condition under which ol would not hold. In the example above, assume further that Options i and p pertain to gains, whereas Options $j$ and q pertain to losses. After Experiments 1 and 2, it could be expected that a positive valence effect would be observed between Options $i$ and $p$, and a negative valence effect would be observed between Options j and q. Thus,

$$
D_{s}(i, p)>D_{i}(i, p) \text { and } D_{s}(j, q)<D_{i}(j, q) \text {. }
$$

Assume further that Option pairs $\mathrm{i}$ and $\mathrm{q}$ are close to each other in their absolute values of payoffs, and so are pairs j and p. In such comparisons, Experiments 1 and 2 showed that it tended to hold that

$$
D_{s}(i, p) \approx D_{i}(j, q) \text { and } D_{i}(i, p) \approx D_{s}(j, q) .
$$

For instance, recall the results in Table 1. For each row in Table 1, this relationship holds if the mean superiority rating in the gain condition equals to the mean inferiority rating in the loss condition; and if the mean 
inferiority rating in the gain condition equals to the mean superiority rating in the loss condition. Empirically, this idea can be tested as a 2 by 2 crossover interaction between domain (gains or losses) and valence (superiority or inferiority) for each gamble pair. For Pairs 1 through 4 in Experiment 1, such interaction terms were highly significant $(\mathrm{F}(1,185)=39.99 * * *$, 23.96***, 24.24*** , 22.75*** for Pairs 1 through 4, respectively (using Bonferroni correction for 4 tests).

Now one can see how positive and negative valence effects may jointly produce violations of OI. Formulas (20) and (21) in combination produce the following prediction:

$$
D_{s}(i, p)>D_{s}(j, q) \text { and } D_{i}(i, p)<D_{i}(j, q) \text {. }
$$

Formulas (22) go contrary to Formulas (19), hence violate OI. Formulas (22) specify the following condition: OI is expected to be violated by intra-valence comparisons of option pairs, one pair of which produces a positive valence effect in the domain of gains, whereas the other pair produces a negative valence effect in the domain of losses.

The right panel in Fig. 6 shows a hypothetical result that follows from a violation of OI. As in the left panel, assume that choice options are rank-ordered by the superiority ratings. The square symbols show that, as the superiority rating increases from the $[\mathrm{j} q]$ pair to the [i p] pair, the corresponding inferiority rating decreases. The deviation from monotonicity shown in the inferiority ratings indicate that OI was violated. As an empirical issue, testing for $\mathrm{Ol}$ requires testing the significance among violations of monotonicity. Such violations need to be detected among superiority ratings when choice options are rank-ordered by inferiority ratings, and among inferiority ratings when the same options are rank-ordered by superiority ratings. Experiment 3 was designed to conduct such analysis.

\section{Method}

Participants. Participants were 438 University of Washington undergraduates. They were enrolled in an introductory psychology course and participated to earn extra credit.

Stimuli. Hypothetical gambles were used as choice options. Sixteen option pairs were generated by having 4 gambles in one set and 4 in another set, and making all possible pairs between the two sets. I refer to one set as Set $A^{\prime \prime}$ and the other as Set $Z^{\prime \prime}$. Consult Appendix $C$ for the gambles. Set $A^{\prime \prime}$ consisted of gambles $\{h, i, j$, $k$ \}, wherein Gambles $h$ and i were exclusively winning and Gambles j and k were exclusively losing. Set $Z^{\prime \prime}$ consisted of gambles $\{0, p, q, r\}$, wherein Gambles o and
TABLE 4

The Stimulus Configuration of Gamble Pairs in Experiment 3

\begin{tabular}{|c|c|c|c|c|c|c|}
\hline & & & \multicolumn{4}{|c|}{ Set Z" gambles } \\
\hline & & & \multicolumn{2}{|c|}{ Gain } & \multicolumn{2}{|c|}{ Loss } \\
\hline & & & 0 & $\mathrm{p}$ & $q$ & $r$ \\
\hline \multirow{3}{*}{$\begin{array}{l}\text { Set } A^{\prime \prime} \\
\text { gambles }\end{array}$} & Gain & $\mathrm{h}$ & [ho] & [h p] & [h q] & {$[\mathrm{hr}$ r] } \\
\hline & & $\mathrm{i}$ & [i o] & [i p] & [i q] & [i r] \\
\hline & Loss & $\begin{array}{l}j \\
k\end{array}$ & 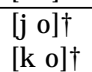 & $\begin{array}{l}{[j \mathrm{p}] \dagger} \\
{[\mathrm{k} \text { p }] \dagger}\end{array}$ & $\left.\begin{array}{l}{[j \mathrm{q}]} \\
{[\mathrm{k}} \\
\mathrm{g}\end{array}\right]$ & $\begin{array}{l}{[\mathrm{j} r]} \\
{[\mathrm{k}} \\
{[\mathrm{r}]}\end{array}$ \\
\hline
\end{tabular}

Note †I ndicates pairs wherein the $Z^{\prime \prime}$ option dominates the $A^{\prime \prime}$ option.

$p$ were exclusively winning and Gambles $q$ and $r$ were exclusively losing. Each element in Set $A^{\prime \prime}$ has a counterpart in Set $Z^{\prime \prime}$ that is created by reversing the sign of the payoff, and vice versa for each element in Set $Z^{\prime \prime}$ having its counterpart in Set $A^{\prime \prime}$. Table 4 shows the configurations of these gamble pairs.

The use of the double quotation in labeling the sets reflects the following ideas: The double quotation indicates that these sets contain different gambles from those used in the previous experiments. More importantly, although Set $A^{\prime \prime}$ gambles tend to dominate Set $Z^{\prime \prime}$ gambles, such a relationship does not hold for every possible gamble pair between Sets $A^{\prime \prime}$ and $Z^{\prime \prime}$. For instance, given a [j o] pair, one would prefer Gamble o to Gamble j.

In Experiment 2, it was necessary that each gamble in Set A was preferred to each gamblein Set $Z$, and that each gamble in Set $A^{\prime}$ was preferred to each gamble in Set $Z^{\prime}$. If some partici pants occasionally preferred a Set $Z$ gamble, then in such a trial the definition of Sets $A$ and $Z$ as determined by preferential ordering must change. Therefore, for such trials, the values of $f\left(A_{\text {good }}\right)$ and $f\left(Z_{\text {good }}\right)$ or $f\left(A_{\text {bad }}\right)$ and $f\left(Z_{\text {bad }}\right)$ in regression analysis must change. To avoid this, the gambles in Experiment 2 were designed so that the preference should be clear from the payoff.

In Experiment 3, however, the critical comparisons involve a subset of option pairs from Set $A^{\prime \prime}$ and $Z^{\prime \prime}$, wherein the paired gambles consist of either exclusively winning or exclusively losing payoffs. These critical comparisons appear in the diagonal cells in Table 4. Although it is possible to detect valence effects with other option pairs, such as [ $j \mathrm{o}$ ] (where the $A^{\prime \prime}$ option is dominated by the $Z^{\prime \prime}$ option), such results would bear few theoretical implications. Nonetheless, data were gathered for such stimulus configurations, because these data contribute to variance estimation in ANOVA with greater reliability (i.e., they supply extra degrees of freedom in calculating $\mathrm{MS}_{\mathrm{e}}$ ). 
Design. A mixed design was used. Between-subjects variables were the levels of the gambles from Set $A^{\prime \prime}$ and Set $Z^{\prime \prime}$ (4 by 4). A within-subject variable was valence of the question (2 levels, superiority or inferiority). Dependent variables were judgments of superiority and inferiority on a 16-point scale, ranging from 0 (no difference) to 15 (maximum difference).

Prediction. The predictions in Experiment 3 were twofold regarding valence effects and OI . First, regarding valence effects, the option pairs that pertain exclusively to gains (the four pairs in the Gain-Gain cell in Table 4) would produce positive valence effects. Conversely, the choice options that pertain exclusively to losses (the four pairs in the Loss-Loss cell in Table 4) would produce negative valence effects. For the remaining eight gamble pairs, no prediction concerning valence effects is drawn from the foregoing argument.

Regarding OI, the following was predicted: If the focus shift model is in effect when positive and negative valence effects are observed, then systematic violations of OI as illustrated in the inequalities Formulas (22) would be observed. Contrarily, if alternative models are in effect in producing valence effects, superiority and inferiority ratings would adhere to OI.

Material and procedure Consult Appendix C for stimuli. Each configuration of stimulus pair was presented to participants, after three practice trials that used similar hypothetical two-outcome gambles. These choice tasks were arranged in one page of a questionnaire, and were distributed to participants along with other filler tasks. Data were gathered in a group setting. For each gamble pair, participants were first asked to express their preferences. Subsequently, each participant provided a superiority rating and an inferiority rating for each pair. The orders of providing superiority and inferiority ratings were randomized and counterbalanced across participants.

\section{Results and Discussion}

Valence ffects. For each gamble pair that pertained to gains, the top half in Table 5 shows the mean superiority rating, mean inferiority rating, t-values, and the number of participants assigned to the pair. Each paired t-test was performed with a Bonferroni correction for four gamble pairs. As predicted, all the gambles showed significantly positive valence effects.

The bottom half in Table 5 shows the mean superiority rating, mean inferiority rating, $t$ values, and the number of participants for each gamble pair that pertained to losses. Each paired t test was performed with a Bonferroni correction for four gamble pairs. Consistent with the prediction, all the significant valence effects were negative. Although the [k q] pair produced
TABLE 5

Positive and Negative Valence Effects Observed in Experiment 3

\begin{tabular}{|c|c|c|c|c|}
\hline Pair label & Superiority & Inferiority & $\mathrm{t}$ value & $n$ \\
\hline \multicolumn{5}{|c|}{ Pairs pertaining exclusively to gains } \\
\hline [h o] & 12.36 & 8.00 & $6.82 * * *$ & 28 \\
\hline [h p] & 13.14 & 6.46 & $7.86 * * *$ & 28 \\
\hline [i o] & 11.92 & 9.20 & $4.42 * * *$ & 25 \\
\hline [i p] & 13.23 & 7.23 & 7.00*** & 26 \\
\hline \multicolumn{5}{|c|}{ Pairs pertaining exclusively to losses } \\
\hline [j q] & 9.52 & 11.93 & $-2.79 *$ & 27 \\
\hline [j r] & 8.39 & 11.75 & $-3.96 * *$ & 28 \\
\hline [k q] & 10.39 & 10.11 & .40 & 25 \\
\hline$[k r]$ & 8.96 & 12.59 & $-3.34^{* *}$ & 27 \\
\hline
\end{tabular}

a positive val ence effect, this result did not reach statistical significance. Thus, in replication of Experiments 1 and 2, Experiment 3 showed that positive valence effects were observed in the domain of gains, whereas negative valence effects were observed in the domain of losses.

Testing for OI. As discussed previously, testing whether Ol is violated requires an examination of pair of gamble pairs, with one of the pairs pertaining to gains and the other pertaining to losses. The diagonal cells in Table 4 show four pairs that pertained exclusively to gains and four that pertained exclusively to Iosses. Thus, Ol can be tested with respect to the $4 \times$ $4=16$ pairs of option pairs. The criterion that qualifies as a violation of Ol is illustrated by using the pair of [i p] and [j q]. Assuming Formulas (22), it would follow that $D_{s}(i, p)>D_{s}(j, q)$ and $D_{i}(i, p)<D_{i}(j, q)$ must simultaneously hold with statistical significance to claim an OI violation. The criterion should be applied for the remaining 15 pairs of option pairs. Hence, for the statistical tests using planned comparisons (explained later), a Bonferroni correction for the Type I error rate was used for 16 (pairs) $\times 2$ (superiority and inferiority ratings) comparisons.

The top and bottom panels of Fig. 7 show the mean superiority and inferiority ratings for all the option pairs used in Experiment 3. The option pairs in the top panel are rank-ordered by the mean superiority ratings, whereas the option pairs in the bottom panel are rank-ordered by the mean inferiority ratings. For the superiority and inferiority ratings, a one-way ANOVA was performed among the 16 cells to calculate $\mathrm{MS}_{\mathrm{e}}$. The omnibus $\mathrm{F}$ statistic and $\mathrm{MS}_{\mathrm{e}}$ were $\mathrm{F}(15,423)$ $=8.94^{* * *}$ and 10.03 for the superiority ratings, respectively. The omnibus F statistic and $\mathrm{MS}_{\mathrm{e}}$ were $\mathrm{F}(15,423)$ $=10.28^{* * *}$ and 13.48 for the inferiority ratings, respectively. 


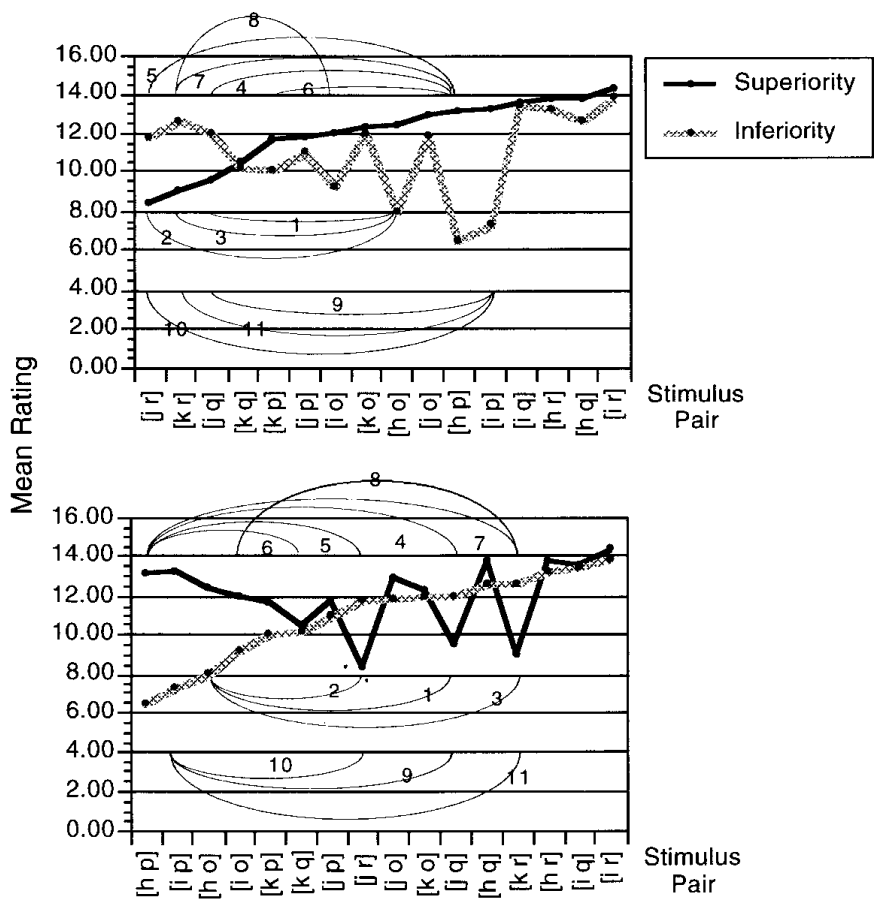

FIG. 7. Mean superiority rating, inferiority rating, and statistically significant violations of OI (indicated by the arc).

Using the $\mathrm{MS}_{\mathrm{e}}$, two planned comparisons were tested regarding the 16 pairs of option pairs that could indicatea violation of OI. For instance, for the [i p] and [ j q] pair, a planned comparison among superiority ratings tested whether $D_{s}(i, p)>D_{s}(j, q)$ and another planned comparison among inferiority ratings tested whether $D_{i}(i, p)<D_{i}(j, q)$. Of 16 pairs of option pairs, 11 showed a significant difference $(\alpha<.05)$ between superiority ratings and a significant difference $(\alpha<.05)$ between inferiority ratings, hence violating Ol. Each pair of them is assigned a number, and connected by an arc in the top and bottom panels of Fig. 7 (e.g., the [i p] and [ $j$ q] pair is assigned a number " 9 "). It is noteworthy that, of 5 pairs of option pairs that did not produce the predicted violations of OI , 4 involved the [k q] pair (no arc is connected to the [k q] pair in Fig. 7). Looking back at Table 5, this is the only gamble pair that failed to produce the predicted negative valence effect. Furthermore, the condition in Formulas (22) assume Formulas (20), i.e., a positive and a negative valence effect are a prerequisite condition to predict a violation of $\mathrm{OI}$. Therefore the failure of the [k q] pair in detecting violations of $\mathrm{Ol}$ is consistent with the condition in Formulas (22).

Thus, it may be claimed that Experiment 3 showed that $\mathrm{OI}$ was violated contingent upon the occurrence of significant positive and negative valence effects (that would supposedly occur with the focus shift: see Experiment 2). This result would go against the alternative explanations to the focus shift model because such accounts require that valence effects occur without violating Ol.

\section{GENERAL DISCUSSION}

This paper extended the line of research set forth by Yamagishi and Miyamoto (1996) in the following sense. Experiment 1 systematically replicated the finding that positive valence effects were observed in the domain of gains, whereas negative valence effects were observed in the domain of losses. Experiment 2 showed that such positive and negative valence effects were associated with systematic focus shift, namely heavy weighting of $A_{\text {good }}$ in superiority judgment and heavy weighting of $Z_{\text {bad }}$ in inferiority judgment. Such a pattern was consistent with the description of valence effects by the focus shift model (Formulas (5) and (6)). Experiment 3 showed that data that exhibited positive and negative valence effects were more in agreement with the focus shift model than with other alternative plausible explanations.

The formalism of the focus shift model shares a kinship with some descriptive models of preferential choice. Particularly, Tversky, Sattath, and Slovic's (1988) contingent weighting model and Shafir's (1993) choice-rejection model commonly rely on feature-set representation of objects of comparison, and the phenomena of interest are explained as reflecting changes in subjective weighting upon these feature sets. In Tversky et al.'s model of preference reversals, each lottery is regarded as consisting of two features, payoff and probability. Reversal of preferential orders between choice and bidding occurs because payoffs are weighted more heavily in bidding than in choice (Tversky, Slovic, \& Kahneman, 1990). Similarly, Shafir's model of the aforementioned choice-rejection asymmetry posits the following subjective weighting process: The choice option consisting of extremely desirable and extremely undesirable features are both chosen and rejected because desirable features are weighted heavily in choice, whereas undesirable features are weighted heavily in rejection. The consequence is that decision makers are provided with reasons to choose the option with extremely desirable features and with reasons to reject the one with extremely undesirable features. It is noteworthy that the formal resemblance among these three model s actualized by no coincidence. They are all inspired by Tversky's (1977) feature-contrast model of asymmetric similarity judgment.

Even in the absence of formalized models, the idea of feature-set representation and subjective weighting has been successfully applied to describe asymmetric psychological phenomena. The Dunning- Parpal asym- 
metry and Dhar and Simonson's (1992) application to consumer choice were al ready introduced. In addition, Houston, Sherman, and Baker (1989) analyzed the effects of stimulus presentation on preferential choice, and their data were explained by differential weighting of the features of the choice options. Suppose that choice options $B$ and $Y$ are presented sequentially in the B-Y order. Suppose further that $B$ is treated as the subject of the comparison, whereas $Y$ is treated as the target of the comparison. Houston et al. hypothesized that the unique features of the subject receive greater weight than the unique features of the target. Therefore, if the unique features of both the subject and the target are desirable, then $B$ should be preferred to $Y$. If the unique features of both the subject and the target are undesirable, then $Y$ should be preferred to $B$. These predictions were supported in experiments using stimuli from a variety of domains.

The focus shift model clearly demarcates desirable and undesirable feature sets, and this approach has its roots in Kahneman and Tversky's (1979) Prospect Theory. The distinction between gains and losses in preference under risk is a key component of Prospect Theory, and this idea is highly analogous to the segregation between desirable and undesirable features. Moreover, the current experiments showed that the direction of valence effects was predictable from the intrinsic pleasantness (gains or losses) of the choice domain. One might find an analogy between these findings and Kahneman and Tversky's demonstration that decision makers switch their attitudes from risk averse to risk seeking between gains and losses. It should be remarked however, that the analogy between the focus shift model and Prospect Theory would not lead to a competition wherein the two models provide different predictions. The reason is that Prospect Theory is a theory of preference, whereas the focus shift model postulates how strength of preference is formed after a clear preferential ordering is established.

Finally, discrepancies from a general principle that is said to direct changes of subjective weights are remarked. As a governing principle, various theorists adopted the notion of "compatibility principle" (e.g., Tversky et al., 1988; Fisher \& Hawkins, 1993; Shafir,
1993). "According to this principle, the weight of any input component is enhanced by its compatibility with the output. The rationale for this principle is that the characteristics of the task and the responsescale prime the most compatible features of the stimulus." (Tversky, Sattath, \& Slovic, 1988, p. 376). It has been claimed that the compatibility principle produces preference reversal phenomena (Tversky, Sattath, \& Slovic, 1989; Tversky, Slovic, \& Kahneman, 1990; Shafir, 1993). Fisher and Hawkins (1993) extended this line of argument and showed that strength of preference ratings were in agreement with the compatibility principle. Back to the current findings, it may seem that the compatibility principle underlies the weighing patterns that are associated with positive and negative valence effects. Concretely, Figure 5 shows that $\alpha_{\mathrm{s}}>$ $\alpha_{\mathrm{i}}$ and $\eta_{\mathrm{s}}<\eta_{\mathrm{i}}$; thus, desirable features were weighted more heavily in superiority judgments, whereas undesirablefeatures were weighted more heavily in inferiority judgments. Moreover, Fig. 4 shows that, in the domain of gains, the focus shift model for superiority judgment produced better fits than the model for inferiority judgment (compare Formulas (7) and (8)). Conversely, in the domain of losses, the model for inferiority judgment produced better fits than the model for superiority judgment (compare F ormulas (9) and (10)). However, caution must be taken before claiming that the compatibility principle explains valence effects, because previous findings (Yamagishi \& Miyamoto, 1996) documented the weighting pattern for positiveand negative valence effects that are not predicted by the compatibility principle. Looking back at Fig. 2, their findings indicate that $\alpha_{\mathrm{s}}>\alpha_{\mathrm{i}}$ and $\eta_{\mathrm{s}}>\eta_{\mathrm{i}}$ when positive valence effects were observed. The compatibility principle has difficulty to predict the $\eta_{\mathrm{s}}>\eta_{\mathrm{i}}$ part, namely heavy weighting of undesirable features in superiority judgment. Likewise, Yamagishi and Miyamoto noted that $\alpha_{\mathrm{s}}<\alpha_{\mathrm{i}}$ and $\eta_{\mathrm{s}}<\eta_{\mathrm{i}}$ when negative valence effects were observed. Again, the $\eta_{\mathrm{s}}<\eta_{\mathrm{i}}$ part is not explained by the compatibility principle. It is not attempted here to claim a boundary condition beyond which the compatibility principle fails to apply. Rather, I am remarking that more research is needed to elaborate the details of psychological mechanisms that produce a variety of asymmetric phenomena.

\section{APPENDIX A: STIMULI USED IN EXPERIMENT 1}

\section{Exclusively Winning Gamble Pairs}

Gamble Pair 1 $A$

Heads

Tails
Win $\$ 80$

Win $\$ 105$
Z

Win $\$ 35$

Win $\$ 60$
Gamble Pair 3

$A$

Win $\$ 95$

Win $\$ 120$
Z

Heads

Tails
Win $\$ 35$

Win $\$ 60$ 
Gamble Pair 2

$A$

Heads

Tails
Win $\$ 80$

Win $\$ 105$
Heads
Tails

Heads
Tails

Gamble Pair 1

Heads

Tails
Z

Win $\$ 50$

Win $\$ 75$
Gamble Pair 4 $A$

Win $\$ 95$

Win $\$ 120$
Z

Win $\$ 50$

Win $\$ 75$

\section{Exclusively Losing Gamble Pairs}

A

Lose $\$ 35$

Lose $\$ 60$

Gamble Pair 2

$$
A \quad Z
$$

Lose $\$ 50$

Lose $\$ 75$
Lose $\$ 80$

Lose $\$ 105$
Gamble Pair 3

$$
\text { A Z }
$$

Lose $\$ 35$

Lose $\$ 95$

Lose $\$ 60$

Lose $\$ 120$

Gamble Pair 4

A Z

Lose $\$ 50$

Lose $\$ 75$

APPENDIX B: STIMULI USED IN EXPERIMENT 2

Gain Condition

\begin{tabular}{|c|c|c|c|c|c|}
\hline & Heads & Tails & & Heads & Tails \\
\hline b & Win $\$ 40$ & Win $\$ 43$ & $\mathrm{v}$ & Win $\$ 15$ & Win $\$ 18$ \\
\hline c & Win $\$ 45$ & Win $\$ 48$ & w & Win $\$ 20$ & Win $\$ 23$ \\
\hline d & Win $\$ 50$ & Win $\$ 53$ & $x$ & Win $\$ 25$ & Win $\$ 28$ \\
\hline e & Win $\$ 55$ & Win $\$ 58$ & $y$ & Win $\$ 30$ & Win $\$ 33$ \\
\hline
\end{tabular}

Set A Gambles

Loss Condition

Set A' Gambles

\begin{tabular}{|c|c|c|c|c|c|}
\hline & Heads & Tails & & Heads & Tails \\
\hline$b^{\prime}$ & Lose $\$ 15$ & Lose $\$ 18$ & $\mathrm{v}^{\prime}$ & Lose $\$ 40$ & Lose $\$ 43$ \\
\hline$c^{\prime}$ & Lose $\$ 20$ & Lose $\$ 23$ & $w^{\prime}$ & Lose $\$ 45$ & Lose $\$ 48$ \\
\hline$d^{\prime}$ & Lose $\$ 25$ & Lose $\$ 28$ & $x^{\prime}$ & Lose $\$ 50$ & Lose $\$ 53$ \\
\hline $\mathrm{e}^{\prime}$ & Lose $\$ 30$ & Lose $\$ 33$ & $y^{\prime}$ & Lose $\$ 55$ & Lose $\$ 58$ \\
\hline
\end{tabular}

Set Z' Gambles

APPENDIX C: STIMULI USED IN EXPERIMENT 3

Set A" Gambles

Set Z" Gambles

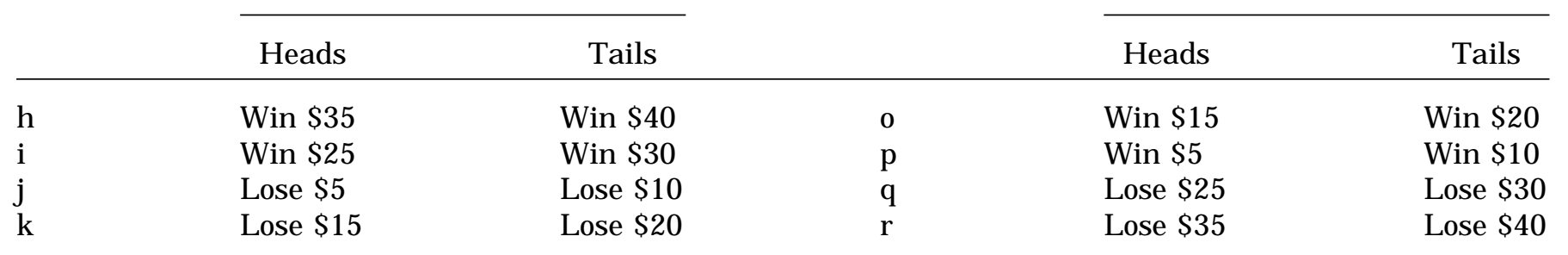




\section{REFERENCES}

Banks, W. P., Clark, H. H., \& Lucy, P. (1975). The locus of the Semantic Congruity Effect in comparative judgments. J ournal of Experimental Psychology: Human Perception and Performance, 104, 35- 47.

Dhar, R., \& Simonson, I. (1992). The effect of the focus of comparison on consumer preference. J ournal of Marketing Research, 29, 430440.

Dunning, D., \& Parpal, M. (1989). Mental addition versus subtraction in counterfactual reasoning: On assessing the impact of personal actions and life events. J ournal of Personality and Social Psychology, 57, 5- 15.

Fisher, G. W., \& Hawkins, S. A. (1993). Strategy compatibility, scale compatibility, and the prominence effects. J ournal of Experimental Psychology: Human Perception and Performance, 19, 580- 597.

Houston, D. A., Sherman, S. J ., \& Baker, S. M. (1989). The influence of unique features and direction of comparison on preferences. J ournal of Experimental Social Psychology, 25, 121- 141.

Kahneman, D., Knetch, J. L., \& Thaler, R. (1990). Experimental Tests of the endowment effect and the Coase Theorem. J ournal of Political Economy, 98, 1325- 1348.

Kahneman, D., Knetch, J . L., \& Thaler, R. H. (1991). The endowment effect, loss aversion, and status quo bias. J ournal of Economic Perspectives, 5, 193- 206.

Kahneman, D., \& Tversky, A. (1979). Prospect Theory: An analysis of decision under risk. Econometrica, 47, 263- 292.

Received: J une 1, 1995
Krantz, D. H., Luce, R. D., Suppes, P., \& Tversky, A. (1971). Foundations of measurement. New York: Academic Press.

Lichtenstein, S., \& Slovic, P. (1971). Reversals of preference between bids and choices in gambling decision. J ournal of Experimental Psychology, 89, 46- 55.

Lindman, H. R. (1971). Inconsistent preferences among gambles. J ournal of Experimental Psychology, 89, 390- 397.

McGill, R., Tukey, J . W., \& Larsen, W. A. (1978). Variations of box plots. The American Statistician, 32, 12- 16.

Shafir, E. (1993). Choosing versus rejecting: Why some options are both better and worse than others. Memory \& Cognition, 21, 546556.

Shafir, E. B., Osherson, D. N., \& Smith, E. E. (1989). An advantage model of choice. J ournal of Behavioral Decision Making, 2, 1-23.

Slovic, P., Lichtenstein, S., \& Fischhoff, B. (1988). Decision Making. In R. C. Atkinson, R. J . Herrnstein, G. Lindzey, \& R. D. Luce (Eds.), Steven's handbook of experimental psychology (pp. 673-738). New York: Wiley.

Tversky, A. (1977). Features of similarity. Psychological Review, 84, 327- 352 .

Tversky, A., Sattath, S., \& Slovic, P. (1988). Contingent weighting in judgment and choice. Psychological Review, 95, 371- 384.

Tversky, A., Slovic, P., \& Kahneman, D. (1990). The causes of preference reversal. American Economic Review, 80, 204- 217.

Yamagishi, K., \& Miyamoto, J . M. (1996). Asymmetries in strength of preference: A focus shift model of valence effects in difference judgment, J ournal of Experimental Psychology: Learning, Memory, and Cognition 22, 493- 509. 